\title{
Nonlinear singularly perturbed integro-differential equations and regularization method
}

\author{
ABDUKHAFIZ BOBODZHANOV \\ National Research University \\ Moscow Power Engineering Institute \\ Krasnokazarmennaya Street 14 \\ 111250, Moscow \\ RUSSIA
}

\author{
BURKHAN KALIMBETOV \\ Ahmet Yassawi University \\ Natural Sciences Institute \\ Sattarkhanov ave. 29 \\ 161200, Turkestan \\ KAZAKHSTAN
}

\author{
VALERY SAFONOV \\ National Research University \\ Moscow Power Engineering Institute \\ Krasnokazarmennaya Street 14, Moscow \\ 111250, Moscow \\ RUSSIA
}

\begin{abstract}
The paper considers a nonlinear integro-differential system of singularly perturbed equations. We discuss the question of the spectrum of its operator, which does not coincide with the spectrum of its limit operator and includes an additionally identically zero point. In the case of linear systems, this difference does not play a special role, since the regularization and construction of the space of solutions of the corresponding iterative problems are realized at nonzero points of the spectrum. In the case of nonlinear problems, the identically zero point of the spectrum plays an essential role in the construction of the solution space in the resonance and nonresonance cases (see below); therefore, in most works using the regularization method in nonlinear problems, only the nonresonance case is usually considered. In the paper, for the classical integrodifferential system, regularization (according to Lomov) is carried out and the corresponding algorithm for constructing asymptotic solutions taking into account the zero point of the spectrum is developed.
\end{abstract}

Key-Words: Singularly perturbed, integro-differential equations, regularization of the integral, iteration problems, normal differential form, main term of asymptotics,

Received: December 15, 2019. Revised: May 12, 2020. Accepted: May 27, 2020. Published: June 8, 2020.

\section{Heuristic considerations}

To understand why, when studying singularly perturbed problems with an integral operator, the zero point of the spectrum should be taken into account, we consider the scalar integro-differential problem

$\varepsilon \frac{d y}{d t}=a(t) y+\int_{0}^{t} k(t, s) y(s, \varepsilon) d s+h(t), y(0, \varepsilon)=y^{0}$.

Denote by $\int_{0}^{t} k(t, s) y(s, \varepsilon) d s=z$. Differentiating this equality by $t$ and multiplying the resulting equation on $\varepsilon$, we obtain the system

$$
\begin{gathered}
\left\{\begin{array}{l}
\varepsilon \frac{d y}{d t}=a(t) y+z+h(t), \\
\varepsilon \frac{d z}{d t}=\varepsilon k(t, t)+\varepsilon \int_{0}^{t} \frac{\partial k(t, s)}{\partial t} y(s, \varepsilon) d s
\end{array} \Leftrightarrow\right. \\
\Leftrightarrow \quad \varepsilon\left(\begin{array}{l}
\frac{d y}{d t} \\
\frac{d z}{d t}
\end{array}\right)=\left(\begin{array}{cc}
a(t) & 1 \\
0 & 0
\end{array}\right)\left(\begin{array}{l}
y \\
z
\end{array}\right)+ \\
+\varepsilon\left(\begin{array}{l}
0 \\
\left.k(t, t)+\int_{0}^{t} \frac{\partial k(t, s)}{\partial t} y(s, \varepsilon) d s\right)+\left(\begin{array}{l}
h(t) \\
0
\end{array}\right) .
\end{array}\right.
\end{gathered}
$$


The matrix of the main part of this system has a spectrum $\quad\left\{\lambda_{1}(t), \lambda_{2}(t)\right\} \equiv\{a(t), 0\}, \quad$ containing identically zero point $\lambda_{2}(t) \equiv 0$ of the spectrum. This point of the spectrum, as is easy to see, arose due to the presence of an integral operator. When constructing a regularized asymptotic solution of linear problems, this point does not play a role, since in linear problems regularization is performed only at nonzero points of the spectrum and the corresponding space of solutions to iterative problems is constructed taking into account only these points of the spectrum (see, for example, [1]). In nonlinear problems, as will be seen from the subsequent discussion, the zero point of the spectrum plays an essential role, especially in the presence of resonances (see below).

\section{Regularization of nonlinear singularly perturbed integro- differential system}

Consider a system

$$
\begin{aligned}
& \varepsilon \frac{d y}{d t}=A(t) y+\int_{0}^{t} K(t, s) y(s, \varepsilon) d s+ \\
& \quad+\varepsilon f(y, t)+h(t), y(0, \varepsilon)=y^{0}, t \in[0, T],
\end{aligned}
$$

where $A(t), K(t, s)$ are the known $(n \times n)$-matrixs, $h(t)=\left\{h_{1}, \ldots, h_{n}\right\}, f(y, t)=\left\{f_{1}, \ldots, f_{n}\right\} \quad$ are known vector functions, $y^{0} \in \mathrm{C}^{n}$ is a known constant vector, $y=y(t) \equiv\left\{y_{1}, \ldots, y_{n}\right\}$ is an unknown vector function, $\varepsilon>0$ is a small parameter. We assume the following conditions are met:

1) $h(t) \in \mathrm{C}^{\infty}\left([0, T], \mathrm{C}^{n}\right), A(t) \in \mathrm{C}^{\infty}\left([0, T], \mathrm{C}^{n \times n}\right)$, $K(t, s) \in \mathrm{C}^{\infty}\left(0 \leq s \leq t \leq T, \mathrm{C}^{n \times n}\right)$;

2) the spectrum $\sigma(A)=\{\lambda(t)\}$ of matrix $A(t)$ satisfies the requirements:

a) $\lambda_{j}(t) \neq 0, \lambda_{i}(t) \neq \lambda_{j}(t), i \neq j, i, j=\overline{1, n}, \forall t \in[0, T]$;

b) $\operatorname{Re} \lambda_{j}(t) \leq 0 \forall t \in[0, T], j=\overline{1, n}$;

3) $f(y, t)$ is polynomial with coefficients $f_{m}(t) \in C^{\infty}\left([0, T], \mathrm{C}^{n}\right), m=\overline{0, N}, N<\infty$;

4) equalities

$$
\sum_{j=1}^{n} m_{j} \lambda_{j}(t)=\lambda_{i}(t), i \in\{1, \ldots, n\}, \sum_{j=1}^{n} m_{j} \lambda_{j}(t)=0
$$

for all multi-indexes with $|m| \equiv \sum_{j=1}^{n} m_{j} \geq 2$ $m_{j} \in Z_{+}, j=\overline{1,4} \quad\left(Z_{+}\right.$is the set of non-negative integers) or do not occur at all $t \in[0, T]$, or are performed identically at all $t \in[0, T]$. Under these conditions we will try to develop algorithm for regularized asymptotic solutions [1] of problem (1). Conditions 4) mean that problem (1) has been investigated in the nonresonance case and in the presence of resonance.

We introduce regularizing variables

$$
\tau_{j}=\frac{1}{\varepsilon} \int_{0}^{t} \lambda_{j}(\theta) d \theta \equiv \frac{\psi_{j}(t)}{\varepsilon}, j=\overline{1, n}
$$

and instead of problem (1) consider the problem

$$
\begin{aligned}
& \frac{\partial \tilde{y}}{\partial t}+\sum_{j=1}^{n} \lambda_{j}(t) \frac{\partial \tilde{y}}{\partial \tau_{j}}-A(t) \tilde{y}-\int_{0}^{t} K(t, s) \tilde{y}\left(s, \frac{\psi(s)}{\varepsilon}, \varepsilon\right) d s- \\
& -\varepsilon f(\tilde{y}, t)=h(t),\left.\tilde{y}(t, \tau, \varepsilon)\right|_{t=0, \tau=0}=y^{0}, t \in[0, T],
\end{aligned}
$$

for the function $\tilde{y}=\tilde{y}(t, \tau, \varepsilon)$, where indicated: $\tau=\left(\tau_{1}, \ldots, \tau_{n}\right), \psi=\left(\psi_{1}, \ldots, \psi_{n}\right)$. It is clear that if $\tilde{y}=\tilde{y}(t, \tau, \varepsilon)$ is the solution of the problem (3) then the vector function $y=\tilde{y}\left(t, \frac{\psi(t)}{\varepsilon}, \varepsilon\right)$ is the exact solution of problem (1), so (3) is the extension of the problem (1). However, it cannot be considered completely regularized, since the integral term $J \tilde{y}=\int_{0}^{t} K(t, s) \tilde{y}\left(s, \frac{\psi(s)}{\varepsilon}, \varepsilon\right) d s$ is not regularized in it. For the regularization of the integral operator it is necessary to introduce a class of asymptotically invariant operator (see [5], p. 62).

We denote

$$
\begin{gathered}
(m, \lambda(t)) \equiv\left(\left(m_{1}, \ldots, m_{n}\right),\left(\lambda_{1}(t), \ldots, \lambda_{n}(t)\right)\right) @ \sum_{j=1}^{n} m_{j} \lambda_{j}(t), \\
(m, \tau) \equiv\left(\left(m_{1}, \ldots, m_{n}\right),\left(\tau_{1}, \ldots, \tau_{n}\right)\right) @ \sum_{j=1}^{n} m_{j} \tau_{j},|m| @ \sum_{j=1}^{n} m_{j}, \\
\Gamma_{j}=\left\{m:(m, \lambda(t)) \equiv \lambda_{j}(t),|m| \geq 2, j=\overline{1, n}\right\}, \\
\Gamma_{0}=\{m:(m, \lambda(t)) \equiv 0,|m| \geq 2\}
\end{gathered}
$$

and we introduce the space $U$ of vector functions $z(t, \tau)$, represented by the sums

$$
\begin{gathered}
z(t, \tau, \sigma)=z_{0}(t)+\sum_{i=1}^{n} z_{i}(t) e^{\tau_{i}}+\sum_{2 \leq|m| \leq N_{z}}^{*} z^{m}(t) e^{(m, \tau)}, \\
m=\left(m_{1}, \ldots, m_{n}\right), z_{i}(t), z^{m}(t) \in C^{\infty}\left([0, T], \mathrm{C}^{n}\right),(4) \\
2 \leq|m| \equiv \sum_{j=1}^{4} m_{j} \leq N_{z},
\end{gathered}
$$

where the asterisk * on the sign of the sum indicates that the summation it only happens on 
the non-resonant multi-indexes, i.e. at $m=\left(m_{1}, \ldots, m_{n}\right) \notin \bigcup_{j=0}^{n} \Gamma_{j}$.

Note that here the degree $N_{z}$ of the polynomial $z(t, \tau)$ relatively exponentials $e^{\tau_{j}}$ depends on element $z$. Also adopted the following terminology: exponents $e^{(m, \tau)}$ with $m=\left(m_{1}, \ldots, m_{4}\right) \in \bigcup_{j=0}^{n} \Gamma_{j}$ are called resonance exponents ; otherwise exponent $e^{(m, \tau)}$ is called nonresonant. We will show that the class $M_{\varepsilon}=\left.U\right|_{\tau=\psi(t) / \varepsilon}$ is asymptotically invariant to the operator $J$.

The image of the operator $J$ on an element (3) of the space $U$ has the form

$$
\begin{gathered}
J z(t, \tau)=\int_{0}^{t} K(t, s) z_{0}(s) d s+ \\
+\sum_{i=1}^{4} \int_{0}^{t} K(t, s) z_{i}(s) e^{\frac{1}{\varepsilon} \int_{0}^{s} \lambda_{i}(\theta) d \theta} d s+ \\
+\sum_{2 \leq m \mid \leq N_{z}}^{*} \int_{0}^{t} K(t, s) z^{m}(s) e^{\frac{1}{\varepsilon} \int_{0}^{s}(m, \lambda(\theta)) d \theta} d s .
\end{gathered}
$$

Integrating by parts, we have

$$
\begin{array}{r}
J_{i}(t, \varepsilon)=\int_{0}^{t} K(t, s) z_{i}(s) e^{\frac{1}{\varepsilon} \int_{0}^{s} \lambda_{i}(s) d \theta} d s= \\
=\varepsilon \int_{0}^{t} \frac{K(t, s) z_{i}(s)}{\lambda_{i}(s)} d e^{\frac{1}{\varepsilon} \int_{0}^{s} \lambda_{i}(s) d \theta}= \\
=\left.\varepsilon \frac{K(t, s) z_{i}(s)}{\lambda_{i}(s)} e^{\frac{1}{\varepsilon} \int_{t_{0}}^{s} \lambda_{i}(\theta) d \theta}\right|_{s=0} ^{s=t}- \\
-\varepsilon \int_{0}^{t}\left(\frac{\partial}{\partial s} \frac{K(t, s) z_{i}(s)}{\lambda_{i}(s)}\right) e^{\frac{1}{\varepsilon} \int_{0}^{s} \lambda_{i}(\theta) d \theta} d s= \\
=\varepsilon\left[\frac{K(t, t) z_{i}(t)}{\lambda_{i}(t)} e^{\frac{1}{\varepsilon} \int_{0}^{t} \lambda_{i}(\theta) d \theta}-\frac{K(t, 0) z_{i}(0)}{\lambda_{i}(0)}\right\rfloor- \\
-\varepsilon \int_{0}^{t}\left(\frac{\partial}{\partial s} \frac{K(t, s) z_{i}(s)}{\lambda_{i}(s)}\right) e^{\frac{1}{\varepsilon} \int_{0}^{s} \lambda_{i}(\theta) d \theta} d s .
\end{array}
$$

Continuing this process next, get the expansion

$$
\begin{gathered}
J_{i}(t, \varepsilon)=\sum_{v=0}^{\infty}(-1)^{v} \varepsilon^{v+1}\left[\left(I_{i}^{v}\left(K(t, s) z_{i}(s)\right)\right)_{s=t} \times\right. \\
\left.\times e^{\left.\frac{1}{\varepsilon} \int_{0}^{t} \lambda_{i}(\theta)\right) d \theta}-\left(I_{i}^{v}\left(K(t, s) z_{i}(s)\right)\right)_{s=0}\right\rfloor,
\end{gathered}
$$

$$
I_{i}^{0}=\frac{1}{\lambda_{i}(s)} . I_{i}^{v}=\frac{1}{\lambda_{i}(s)} I_{i}^{v-1}(v \geq 1, i=\overline{1, n}) .
$$

Applying the operation of integration by parts to the integrals

$$
J_{m}(t, \varepsilon)=\int_{0}^{t} K(t, s)(s) e^{\frac{1}{\varepsilon} \int_{0}^{s}(m, \lambda(\theta)) d \theta} d s,
$$

will have:

$$
\begin{gathered}
J_{m}(t, \varepsilon)=\int_{0}^{t} K(t, s) z^{m}(s) e^{\frac{1}{\varepsilon} \int_{0}^{s}(m, \lambda(\theta)) d \theta} d s= \\
=\varepsilon \int_{0}^{t} \frac{K(t, s) z^{m}(s)}{(m, \lambda(s))} d e^{\frac{1}{\varepsilon} \int_{0}^{s}(m, \lambda(\theta)) d \theta}= \\
=\varepsilon\left[\frac{K(t, s) z^{m}(s)}{(m, \lambda(s))} e^{\frac{1}{\varepsilon} \int_{0}^{t}(m, \lambda(\theta)) d \theta}-\frac{K(t, 0) z^{m}(0)}{(m, \lambda(0))} \mid-\right. \\
-\varepsilon \int_{t_{0}}^{t}\left(\frac{\partial}{\partial s} \frac{K(t, s) z^{m}(s)}{(m, \lambda(s))}\right) e^{\frac{1}{\varepsilon} \int_{0}^{s}(m, \lambda(\theta)) d \theta} d s= \\
=\sum_{v=0}^{\infty}(-1)^{v} \varepsilon^{v+1}\left[\left(I_{m}^{v}\left(K(t, s) z^{m}(s)\right)\right) \times\right. \\
\left.\times e^{\frac{1}{\varepsilon} \int_{0}^{t}(m, \lambda(\theta)) d \theta}-\left(I_{m}^{v}\left(K(t, s) z^{m}(s)\right)\right)_{s=0}\right], \\
I_{m}^{0}=\frac{1}{(m, \lambda(s))} ., I_{m}^{v}=\frac{1}{(m, \lambda(s))} \frac{\partial}{\partial s} I_{m}^{v-1}(v \geq 1,|m| \geq 2),
\end{gathered}
$$

(it is taken into account that for the elements of the space $U$ the inequalities $(m, \lambda(s)) \neq 0$ are satisfied, because $\left.\left.m \notin \Gamma_{0}\right)\right)$. So, the image of the operator $J$ on element (3) of the space $U$ is represented in the form of a series

$$
\begin{gathered}
J z(t, \tau)=\int_{0}^{t} K(t, s) z_{0}(s) d s+ \\
+\sum_{i=1}^{n} \sum_{v=0}^{\infty}(-1)^{v} \varepsilon^{v+1}\left\lfloor\left(I_{i}^{v}\left(K(t, s) z_{i}(s)\right)\right)_{s=t} e^{\left.\frac{1}{\varepsilon} \int_{0}^{t} \lambda_{i}(\theta)\right) d \theta}-\right. \\
\left.-\left(I_{i}^{v}\left(K(t, s) z_{i}(s)\right)\right)_{s=0}\right\rfloor+\sum_{2 \leq|m| \leq N_{z}}^{*} \sum_{v=0}^{\infty}(-1)^{v} \varepsilon^{v+1} \times \\
\times\left\lfloor\left(I_{m}^{v}\left(K(t, s) z^{m}(s)\right)\right)_{s=t} e^{\frac{1}{\varepsilon} \int_{0}^{t}(m, \lambda(\theta)) d \theta}-\right. \\
\left.-\left(I_{m}^{v}\left(K(t, s) z^{m}(s)\right)\right)_{s=0}\right\rfloor, v \geq 1 .
\end{gathered}
$$

It is easy to show (see [2], pp. 291-294) that this series converges asymptotically as $\varepsilon \rightarrow+0$ (uniformly in $t \in[0, T]$ ). This means that the 
class $M_{\varepsilon}$ of asymptotically invariant (as $\varepsilon \rightarrow+0$ ) relative to the operator $J$. Let us introduce operators $R_{v}: U \rightarrow U$, acting on each element $z(t, \tau) \in U$ of the form (3) according to the law:

$$
\begin{gathered}
R_{0} z(t, \tau)=\int_{0}^{t} K(t, s) z_{0}(s) d s, \\
R_{1} z(t, \tau)=\sum_{i=1}^{n}\left[\left(I_{i}^{0}\left(K(t, s) z_{i}(s)\right)\right)_{s=t} e^{\tau_{i}}-\right. \\
\left.-\left(I_{i}^{0}\left(K(t, s) z_{i}(s)\right)\right)_{s=0}\right\rfloor+ \\
+\sum_{2 \leq|m| \leq N_{z}}^{*}\left[\left(I_{m}^{0}\left(K(t, s) z^{m}(s)\right)\right)_{s=t} e^{(m, \tau)}-\right. \\
\left.-\left(I_{m}^{0}\left(K(t, s) z^{m}(s)\right)\right)_{s=0}\right], \\
R_{v+1} z(t, \tau)=\sum_{i=1}^{n}(-1)^{v} \varepsilon^{v+1}\left[\left(I_{i}^{v}\left(K(t, s) z_{i}(s)\right)\right)_{s=t} e^{\tau_{i}}-\right. \\
\left.-\left(I_{i}^{v}\left(K(t, s) z_{i}(s)\right)\right)_{s=0}\right\rfloor+ \\
+\sum_{v=0}^{*}(-1)^{v} \varepsilon^{v+1}\left[\left(I_{m}^{v}\left(K(t, s) z^{m}(s)\right)\right)_{s=t} e^{(m, \tau)}-\right. \\
\left.-\left(I_{m}^{v}\left(K(t, s) z^{m}(s)\right)\right)_{s=0}\right\rfloor, v \geq 1 .
\end{gathered}
$$

Let now $\tilde{y}(t, \tau, \varepsilon)$ is an arbitrary continuous on $(t, \tau) \in[0, T] \times\left\{\tau: \operatorname{Re} \tau_{j} \leq 0, j=\overline{1, n}\right\}$ function with asymptotic expansion

$$
\tilde{y}(t, \tau, \varepsilon)=\sum_{k=0}^{\infty} \varepsilon^{k} y_{k}(t, \tau), y_{k}(t, \tau) \in U,
$$

which converges (when $\varepsilon \rightarrow+0$, uniformly for $\left.(t, \tau) \in[0, T] \times\left\{\tau: \operatorname{Re} \tau_{j} \leq 0, j=\overline{1, n}\right\}\right)$. Then the image of the operator $J$ on this function is decomposed in asymptotical series

$$
J \tilde{y}(t, \tau, \varepsilon)=\sum_{k=0}^{\infty} \varepsilon^{k} J y_{k}(t, \tau)=\left.\sum_{r=0}^{\infty} \varepsilon^{r} \sum_{s=0}^{r} R_{r-s} y_{s}(t, \tau)\right|_{\tau=\psi(t) / \varepsilon} .
$$

This equality is the basis for the introduction of the extension of an operator $J$ on series of the form (6):

$$
\tilde{J} \tilde{y}(t, \tau, \varepsilon) \equiv \tilde{J}\left(\sum_{k=0}^{\infty} \varepsilon^{k} y_{k}(t, \tau)\right) @ \sum_{r=0}^{\infty} \varepsilon^{r} \sum_{s=0}^{r} R_{r-s} y_{s}(t, \tau) .
$$

Although the operator $\tilde{J}$ is defined formally, its usefulness is obvious, as in practice usually build $N$ - th approximation of the asymptotic solution (1), which will involve only partial sums of the series (6), having no formal, but true meaning. Now we can write down a problem that is completely regularized with respect to the original problem (1):

$$
\begin{aligned}
& \varepsilon \frac{\partial \tilde{y}}{\partial t}+\sum_{j=1}^{n} \lambda_{j}(t) \frac{\partial \tilde{y}}{\partial \tau_{j}}-A(t) \tilde{y}-\varepsilon f(\tilde{y}, t)- \\
& -\tilde{J} \tilde{y}=h(t),\left.\quad \tilde{y}(t, \tau, \varepsilon)\right|_{t=0, \tau=0}=y^{0}, t \in[0, T] .
\end{aligned}
$$

\section{Iterative problems and their solvability in the space $U$. The solution of the first iteration problem}

Substituting the series (6) into (8) and equating the coefficients of equal powers of $\varepsilon$, we get the following iterative problems:

$$
\begin{gathered}
\mathrm{L} y_{0}(t, \tau) \equiv \sum_{j=1}^{n} \lambda_{j}(t) \frac{\partial y_{0}}{\partial \tau_{j}}-A(t) y_{0}-R_{0} y_{0}= \\
=h(t), \quad y_{0}(0,0)=y^{0} \\
\begin{aligned}
\mathrm{L} y_{1}(t, \tau)=-\frac{\partial y_{0}}{\partial t}+ & f\left(y_{0}, t\right)+R_{1} y_{0}, y_{1}(0,0)=0 \\
\mathrm{~L} y_{2}(t, \tau)=-\frac{\partial y_{1}}{\partial t}+ & \frac{\partial f\left(y_{0}, t\right)}{\partial y} y_{1}+R_{1} y_{1}+ \\
& +R_{2} y_{0}, y_{2}(0,0)=0
\end{aligned}
\end{gathered}
$$

$$
\begin{aligned}
& \mathrm{L} y_{k}(t, \tau)=-\frac{\partial y_{k-1}}{\partial t}+P_{k}\left(y_{0}, \ldots, y_{k-1}, t\right)+ \\
& \quad+R_{k} y_{0}+\ldots+R_{1} y_{k-1}, y_{k}(0,0)=0, k \geq 1,
\end{aligned}
$$

where $P_{k}\left(y_{0}, \ldots, y_{k-1}, t\right)$ are some polynomials in $y_{1}, \ldots, y_{k-1}$ with coefficients that depend on the partial derivatives of the function $f(y, t)$ at the point $y=y_{0}(t, \tau)$.

Each of the iterative problems $\left(9_{k}\right)$ can be written in the form

$$
\begin{array}{r}
\mathrm{L} z(t, \tau) \equiv \sum_{j=1}^{n} \lambda_{j}(t) \frac{\partial z}{\partial \tau_{j}}-A(t) z-R_{0} z= \\
=H(t, \tau), \quad z(0,0)=z^{*},
\end{array}
$$

where $H(t, \tau)=H_{0}(t)+\sum_{i=1}^{n} H_{i}(t)^{\tau_{i}}+\sum_{2 \leq|m| \leq N_{z}}^{*} H^{m}(t) e^{(m, \tau)}$ is a known vector function of the space $U, z^{*}$ is a known constant in a complex vector space $\mathrm{C}^{n}$, and the operator $R_{0}$ has the form (see $\left(5_{0}\right)$ )

$$
\begin{gathered}
R_{0} z \equiv R_{0}\left(z_{0}(t)+\sum_{i=1}^{n} z_{i}(t) e^{\tau_{i}}+\sum_{2 \leq|m| \leq N_{z}}^{*} z^{m}(t) e^{(m, \tau)}\right) @ \\
@ \int_{0}^{t} K(t, s) z_{0}(s) d s .
\end{gathered}
$$


In the future, we need $\lambda_{j}(t)$-eigenvectors $\varphi_{j}(t)$ of a matrix $A(t)$ and $\bar{\lambda}_{j}(t)$-eigenvectors $\chi_{j}(t)$ of a matrix $A^{*}(t)$. These vectors we take biorthonormalized, i.e.

$$
\left(\varphi_{k}(t), \chi_{j}(t)\right)=\left\{\begin{array}{l}
1, k=j, \\
0, k \neq j
\end{array}(k, j=\overline{1, n}) .\right.
$$

We introduce a scalar product (for each $t \in[0, T]$ ) in the space $U$ :

$$
\begin{aligned}
<z, w> & \equiv<z_{0}(t)+\sum_{i=1}^{n} z_{i}(t) e^{\tau_{i}}+\sum_{2 \leq|m| \leq N_{z}}^{*} z^{m}(t) e^{(m, \tau)}, \\
w_{0}(t) & +\sum_{i=1}^{n} w_{i}(t) e^{\tau_{i}}+\sum_{2 \leq|m| \leq N_{w}}^{*} w^{m}(t) e^{(m, \tau)}>@ \\
@ & \left(z_{0}(t), w_{0}(t)\right)+\sum_{i=1}^{n}\left(z_{i}(t), w_{i}(t)\right)+ \\
& +\sum_{2 \leq|m| \leq \min \left(N_{z}, N_{w}\right)}^{*}\left(z^{m}(t), w^{m}(t)\right),
\end{aligned}
$$

where $(*, *)$ is the usual scalar product in a complex space $\mathrm{C}^{n}$. We prove the following statement.

Theorem 1. Let the conditions 1)-4) hold true and the right part $H(t, \tau)=H_{0}(t)+\sum_{i=1}^{n} H_{i}(t) e^{\tau_{i}}+\sum_{2 \leq|m| \leq N_{z}}^{*} H^{m}(t) e^{(m, \tau)}$ of (10) belongs to the space $U$. Then the system (10) is solvable in $U$, if and only if

$$
<H(t, \tau), \chi_{j}(t) e^{\tau_{j}}>\equiv 0, j=\overline{1, n}, \forall t \in[0, T] .
$$

Proof. We define a solution of (10) as a member (4) of the space $U$ :

$$
z(t, \tau)=z_{0}(t)+\sum_{i=1}^{n} z_{i}(t) e^{\tau_{i}}+\sum_{2 \leq|m| \leq N_{z}}^{*} z^{m}(t) e^{(m, \tau)},
$$

Substituting (12) into (10) we have

$$
\begin{gathered}
\sum_{i=1}^{n}\left[\lambda_{i}(t) I-A(t)\right] z_{i}(t) e^{\tau_{i}}+ \\
+\sum_{2 \leq|m| \leq N_{z}}^{*}[(m, \lambda(t)) I-A(t)] z^{m}(t) e^{(m, \tau)}- \\
-A(t) z_{0}(t)-\int_{0}^{t} K(t, s) z_{0}(t) d s= \\
=H_{0}(t)+\sum_{i=1}^{n} H_{i}(t) e^{\tau_{i}}+\sum_{2 \leq|m| \leq N_{z}}^{*} H^{m}(t) e^{(m, \tau)} .
\end{gathered}
$$

Equating here separately free members and coefficients of the same exponents, we obtain the following equations:

$$
\begin{gathered}
-A(t) z_{0}(t)-\int_{0}^{t} K(t, s) z_{0}(t) d s=H_{0}(t), \\
{\left[\lambda_{i}(t) I-A(t)\right] z_{i}(t)=H_{i}(t), i=\overline{1, n}}
\end{gathered}
$$

$$
\begin{aligned}
& {[(m, \lambda(t)) I-A(t)] z^{m}(t)=H^{m}(t),} \\
& 2 \leq|m| \leq N_{H}, m \notin \bigcup_{j=0}^{n} \Gamma_{j} .
\end{aligned}
$$

Since the matrix $A(t)$ is invertible, the system (13) can be written as

$$
z_{0}(t)=\int_{t_{0}}^{t}\left(-A^{-1}(s) K(t, s)\right) z_{0}(s) d s-A^{-1}(t) H_{0}(t) .
$$

Due to the smoothness of the kernel $-A^{-1}(t) K(t, s)$ and heterogeneity $-A^{-1}(t) H_{0}(t)$ this integral Volterra system has a unique solution $z_{0}(t) \in C^{\infty}\left([0, T], \mathrm{C}^{n}\right)$. Systems $\left(14_{m}\right)$ also have unique solutions

$$
z^{m}(t)=[(m, \lambda(t)) I-A(t)]^{-1} H^{m}(t),
$$

because $m \notin \bigcup_{j=1}^{n} \Gamma_{j}$. Systems $\left(14_{i}\right)$ have been solved in the space $C^{\infty}\left([0, T], \mathrm{C}^{n}\right)$ if and only if the following identities $\quad\left(H_{i}(t), \chi_{i}(t)\right) \equiv 0 \forall t \in[0, T]$, $i=\overline{1, n}$ are performed. It is easy to see that these identities coincide with the identities (11). Thus, the condition (11) is necessary and sufficient for the solubility of the system (10) in the space $U$. The theorem is proved.

Remark 1. If identity (11) holds, then under conditions 1)-4), system (10) has the following solution in the space $U$ :

$$
z(t, \tau)=z_{0}(t)+\sum_{i=1}^{n} z_{i}(t) e^{\tau_{i}}+\sum_{2 \leq|m| \leq N_{z}}^{*} z^{m}(t) e^{(m, \tau)}
$$

where indicated:

$$
\begin{gathered}
z_{k}(t) \equiv\left(\alpha_{k}(t) \varphi_{k}(t)+\sum_{s=1, s \neq k}^{n} \frac{\left(H_{k}(t), \chi_{s}(t)\right)}{\lambda_{k}(t)-\lambda_{s}(t)} \varphi_{s}(t)\right) e^{\tau_{k}}, \\
k=\overline{1, n}, \\
z^{m}(t) \equiv[(m, \lambda(t)) I-A(t)]^{-1} H^{m}(t), \\
2 \leq|m| \leq N_{H}, \quad m \notin \bigcup_{j=0}^{n} \Gamma_{j} ;
\end{gathered}
$$

at the same time $\alpha_{k}(t) \in C^{\infty}\left([0, T], \mathrm{C}^{n}\right)$ are arbitrary functions, $k=1,2$, and $z_{0}(t)$ is the solution of the integrated system $\left(14_{0}\right)$.

\section{The construction solutions of iterative problems}

We proceed to the construction of solutions of iterative problems $\left(9_{k}\right)$ in space $U$. We will not formulate a theorem on the unique solvability of the 
general problem (10). We show that the application of Theorem 1 to two consecutive iteration problems $\left(9_{k}\right)$ and $\left(9_{k+1}\right)$, allows you to determine the solution of the first of them uniquely in the class $U$. First note that the systems $\left(9_{k+1}\right)$ have the form

$$
\begin{aligned}
\mathrm{L} y_{k+1}(t, \tau) & =-\frac{\partial y_{k}}{\partial t}+P_{k}\left(y_{0}, \ldots, y_{k}, t\right)+ \\
& +R_{k+1} y_{0}+\ldots+R_{1} y_{k}, \quad k \geq 0,
\end{aligned}
$$

where $y_{0}(t, \tau), \ldots, y_{k}(t, \tau)$ are the solutions of previous problems $\left(9_{0}\right), \ldots,\left(9_{k}\right)$ in the space $U$. Right-hand side of this system:

$$
H(t, \tau) \equiv-\frac{\partial y_{k}}{\partial t}+P_{k}\left(y_{0}, \ldots, y_{k}, t\right)+R_{k+1} y_{0}+\ldots+R_{1} y_{k}
$$

can not belong to the space $U$, as the vector function $P_{k}\left(y_{0}, \ldots, y_{k}, t\right)$ may contain a resonance exponents $e^{(m, \tau)}$ with multi-indexes $m \in \bigcup_{j=0}^{n} \Gamma_{j}$, and so the right side $H(t, \tau)$ of the system (17) belongs, generally speaking, the space $V$ of vector functions $z(t, \tau)$, written as the sum

$$
\begin{gathered}
z(t, \tau, \sigma)=z_{0}(t)+\sum_{i=1}^{n} z_{i}(t) e^{\tau_{i}}+\sum_{2 \leq m \mid \leq N_{z}} z^{m}(t) e^{(m, \tau)}, \\
m=\left(m_{1}, \ldots, m_{n}\right), z_{i}(t), z^{m}(t) \in C^{\infty}\left([0, T], \mathrm{C}^{n}\right), \\
2 \leq|m| \equiv \sum_{j=1}^{n} m_{j} \leq N_{z},
\end{gathered}
$$

which may contain resonant exponents. Then the well-known theory (see. [1], p. 234) it is necessary to make an embedding $H(t, \tau) \rightarrow H(t, \tau)$ into space $U$. Makes it so. We denote by $\hat{z}(t, \tau)\left(\right.$ or $\left.(z(t, \tau))^{\wedge}\right)$ the restriction element $z(t, \tau) \in V$ at $\tau=\frac{\psi(t)}{\varepsilon}$, i. e. $\left.\hat{z}(t, \tau) \equiv z(t, \tau)\right|_{\tau=\frac{\psi(t)}{\varepsilon}} . \quad$ The operation $\wedge: z(t, \tau) \rightarrow \hat{z}(t, \tau), \quad$ of assigning to each element $z(t, \tau)$ of the space $V$ element $\hat{z}(t, \tau)$ of the space $U$ such that $\left.\hat{z}(t, \tau) \equiv z(t, \tau)\right|_{\tau=\frac{\psi(t)}{\varepsilon}}$, we call operation of the embedding $V$ into the space $U$. For example, if $\lambda_{1}(t)=-i \omega(t), \lambda_{2}(t)=+i \omega(t)$, then terms of the space $V$ with exponents $e^{(s+1) \tau_{1}+s \tau_{2}}, e^{s \tau_{1}+(s+1) \tau_{2}}, e^{s \tau_{1}+s \tau_{2}}$ are resonant and their embeddings move in terms exponents $\quad e^{\tau_{1}}, e^{\tau_{2}}, e^{0}=1, \quad$ since $\left(e^{(s+1) \tau_{1}+s \tau_{2}}\right)^{\wedge}=e^{\tau_{1}},\left(e^{s \tau_{1}+(s+1) \tau_{2}}\right)^{\wedge}=\quad=e^{\tau_{2}}$, $\left(e^{s \tau_{1}+s \tau_{2}}\right)^{\wedge}=e^{0}=1$. We embed the right part of the iterative problems into space $U$, i. e. replace problems $\left(9_{k}\right)$ to problems

$$
\begin{aligned}
& \mathrm{L} y_{0}(t, \tau) \equiv \sum_{j=1}^{n} \lambda_{j}(t) \frac{\partial y_{0}}{\partial \tau_{j}}-A(t) y_{0}-R_{0} y_{0}= \\
& =h(t), \quad y_{0}(0,0)=y^{0} \text {; } \\
& \mathrm{L} y_{1}(t, \tau)=-\frac{\partial y_{0}}{\partial t}+\hat{f}\left(y_{0}, t\right)+R_{1} y_{0}, y_{1}(0,0)=0 \text {; } \\
& \mathrm{L} y_{2}(t, \tau)=-\frac{\partial y_{1}}{\partial t}+\left(\frac{\partial f\left(y_{0}, t\right)}{\partial y} y_{1}\right)^{\wedge}+R_{1} y_{1}+ \\
& +R_{2} y_{0}, y_{2}(0,0)=0 \text {; } \\
& \mathrm{L} y_{k}(t, \tau)=-\frac{\partial y_{k-1}}{\partial t}+\hat{P}_{k}\left(y_{0}, \ldots, y_{k-1}, t\right)+ \\
& +R_{k} y_{0}+\ldots+R_{1} y_{k-1}, y_{k}(0,0)=0, k \geq 1
\end{aligned}
$$

(images of linear operators $\frac{\partial}{\partial t}$ and $R_{k}$ do not need an embedding to the space $U$, as these operators act from $U$ to $U$ ). On the construction of an asymptotic solution of the original problem (1) such embedding will not be affected, as in the narrowing $\tau=\frac{\psi(t)}{\varepsilon}$ of the series of problems $\left(\overline{9}_{k}\right)$ will coincide with a series of problems $\left(\overline{9}_{k}\right)$ (see. [1], c. 234-235]).

Using Theorem 1, we will try to find a solution of the first iteration of the problem $\left(\overline{9}_{0}\right)$. Since the right side $h(t)$ of the system $\left(\overline{9}_{0}\right)$ satisfies the condition (11), this system has (according to (15)(16)) the solution in the space $U$ in the form (note that in all $\left(\overline{9}_{0}\right) \quad H_{j}(t) \equiv 0, H^{m}(t) \equiv 0, j=\overline{1, n}$, $|m| \geq 2$ )

$$
y_{0}(t, \tau)=y_{0}^{(0)}(t)+\sum_{k=1}^{2} \alpha_{k}^{(0)}(t) \varphi_{k}(t) e^{\tau_{k}},
$$

where $y_{0}^{(0)}(t)$ is a solution of the integral system

$$
y_{0}^{(0)}(t)=\int_{0}^{t}\left(-A^{-1}(s) K(t, s)\right) y_{0}^{(0)}(s) d s-A^{-1}(t) h(t),
$$

where $\quad \alpha_{k}^{(0)}(t) \in C^{\infty}\left(\left[t_{0}, T\right], \mathrm{C}^{1}\right) \quad$ are arbitrary functions, $k=\overline{1, n}$. Subordinating (18) to the initial condition $y_{0}(0,0)=y^{0}$, we have

$$
\begin{aligned}
& y_{0}^{(0)}(0)+\sum_{k=1}^{2} \alpha_{k}^{(0)}(0) \varphi_{k}(0)=y^{0} \Leftrightarrow \\
\Leftrightarrow & \sum_{k=1}^{2} \alpha_{k}^{(0)}(0) \varphi_{k}(0)=y^{0}+A^{-1}(0) h(0) .
\end{aligned}
$$


Multiplying this equality scalarly by $\chi_{j}(0)$ and taking into account biorthogonality of systems $\left\{\varphi_{k}(t)\right\}$ and $\left\{\chi_{j}(t)\right\}$, we find the value $\alpha_{k}^{(0)}(0)=\left(z^{0}+A^{-1}(0) h(0), \chi_{k}(0)\right), k=\overline{1, n}$. For a full evaluation of the functions $\alpha_{k}^{(0)}(t)$ move to the next iteration problems $\left(\overline{9}_{1}\right)$. Substituting the solution (18) of the system $\left(\overline{9}_{0}\right)$ into it, we arrive at the following system:

$$
\begin{aligned}
& \mathrm{L} y_{1}(t, \tau)=-\frac{d}{d t} y_{0}^{(0)}(t)-\sum_{k=1}^{n} \frac{d}{d t}\left(\alpha_{k}^{(0)}(t) \varphi_{k}(t)\right) e^{\tau_{k}}+ \\
& +\left(f\left(y_{0}^{(0)}(t)+\sum_{k=1}^{n} \alpha_{k}^{(0)}(t) \varphi_{k}(t) e^{\tau_{k}}, t\right)\right)^{\wedge}+ \\
& +\sum_{j=1}^{n}\left[\frac{\left(K(t, t) \alpha_{j}^{(0)}(t) \varphi_{j}(t)\right)}{\lambda_{j}(t)} e^{\tau_{j}}-\frac{\left(K\left(t, t_{0}\right) \alpha_{j}^{(0)}(0) \varphi_{j}(0)\right)}{\lambda_{j}(0)}\right]
\end{aligned}
$$

(here we have used the expression $\left(5_{1}\right)$ for $R_{1} z(t, \tau)$ and take into account that when $z(t, \tau)=y_{0}(t, \tau)$ the sum $\left(5_{1}\right)$ remain only terms with $\left.\left(5_{1}\right)\right)$. Taylor's formula (see. [2], p. 95), we have

$$
\begin{gathered}
f\left(t, y_{0}(t, \tau)\right)=f\left(y_{0}^{(0)}(t), t\right)+ \\
+\sum_{1 \leq|m| \leq N}\left(\frac{1}{|m| !} \sum_{j=1}^{n} \frac{\partial f\left(t, y_{0}^{(0)}(t)\right)}{\partial y} \varphi_{j}(t) \alpha_{j}^{(0)}(t) e^{\tau_{j}}\right)^{[[m]]} \equiv \\
\equiv f\left(y_{0}^{(0)}(t), t\right)+\sum_{j=1}^{n}\left(\frac{\partial f\left(t, y_{0}^{(0)}(t)\right)}{\partial y} \varphi_{j}(t) \alpha_{j}^{(0)}(t)\right) e^{\tau_{j}}+ \\
+\sum_{j=0}^{n} \sum_{m^{j} \in \Gamma_{j}, m^{m^{j}} \mid \leq N}\left(\tilde{f}^{\left(m^{j}\right)}(t)\left(\alpha^{(0)}(t)\right)^{m^{j}} e^{\left(m^{j}, \tau\right)}+\right. \\
+\sum_{m \notin \bigcup_{j=0}^{n} \Gamma_{j}, 2 \leq|m| \leq N} \tilde{f}^{m}(t)\left(\alpha^{(0)}(t)\right)^{(m, \tau)},
\end{gathered}
$$

where $\alpha^{(0)}(t) \equiv\left(\alpha_{1}^{(0)}(t), \ldots, \alpha_{n}^{(0)}(t)\right), \quad \tilde{f}^{\left(m^{j}\right)}(t), \tilde{f}^{(m)}(t) \in$ $\in \mathrm{C}^{\infty}\left([0, T], C^{n}\right)$ are known vector functions, $|m| \geq 2$. Producing an embedding vector function $f\left(t, y_{0}(t, \tau)\right)$ in space $U$, we rewrite system (20) as a

$$
\begin{aligned}
\mathrm{L} y_{1}(t, \tau)= & -\frac{d}{d t} y_{0}^{(0)}(t)-\sum_{j=1}^{n}\left[\left(\alpha_{j}^{(0)}(t) \varphi_{j}(t)\right)^{\cdot} e^{\tau_{j}}-\right. \\
& -\frac{\partial f\left(t, y_{0}^{(0)}(t)\right)}{\partial y} \varphi_{j}(t) \alpha_{j}^{(0)}(t) e^{\tau_{j}}-
\end{aligned}
$$

$$
\begin{aligned}
& \left.-\sum_{j=0}^{n} \sum_{m^{j} \in \Gamma_{j}, m^{j} \mid \leq N} \tilde{f}^{\left(m^{j}\right)}(t)\left(\alpha^{(0)}(t)\right)^{m^{j}} e^{\tau_{j}}\right\rfloor+f\left(y_{0}^{(0)}(t), t\right)+ \\
& +\sum_{m \notin \bigcup_{j=0}^{n} \Gamma_{j}, 2 \leq|m| \leq N} \tilde{f}^{(m)}(t)\left(\alpha^{(0)}(t)\right)^{m} e^{(m, \tau)}+\quad(21) \\
& +\sum_{j=1}^{n}\left\lfloor\frac{\left(K(t, t) \alpha_{j}^{(0)}(t) \varphi_{j}(t)\right)}{\lambda_{j}(t)} e^{\tau_{j}}-\frac{\left(K\left(t, t_{0}\right) \alpha_{j}^{(0)}(0) \varphi_{j}(0)\right)}{\lambda_{j}(0)}\right\rfloor
\end{aligned}
$$

Here we have introduced the notation $\tau_{0}=0$ and used the identity

$$
\begin{gathered}
\left(\tilde{f}^{(m)}(t)\left(\alpha^{(0)}(t)\right)^{m^{j}} e^{\left(m^{j}\right)}\right)^{\wedge} \equiv \\
\equiv \tilde{f}^{(m)}(t)\left(\alpha^{(0)}(t)\right)^{m^{j}}\left(e^{m^{j}}\right)^{\wedge}=\tilde{f}^{\left(m^{j}\right)}(t)\left(\xi^{(0)}(t)\right)^{m^{j}} e^{\tau_{j}} .
\end{gathered}
$$

Now right-hand side $H(t, \tau)$ of (21) belongs to $U$.

The orthogonality conditions (11) gives:

$$
\begin{gathered}
<H(t, \tau), \chi_{j}(t) e^{\tau_{j}}>\equiv 0 \quad(j=\overline{1, n}) \Leftrightarrow \\
\Leftrightarrow-\dot{\alpha}_{j}^{(0)}-\left(\dot{\varphi}_{j}(t), \chi_{j}(t)\right) \alpha_{j}^{(0)}+\frac{\left(K(t, t) \alpha_{j}^{(0)}(t) \varphi_{j}(t)\right)}{\lambda_{j}(t)}+ \\
+\frac{\partial f\left(t, y_{0}^{(0)}(t)\right)}{\partial y}\left(\varphi_{j}(t), \chi_{j}(t)\right) \alpha_{j}^{(0)}+ \\
+\sum_{m^{j} \in \Gamma_{j}, m^{j} \mid \leq N}\left(\tilde{f}^{\left(m^{j}\right)}(t), \chi_{j}(t)\right)\left(\xi^{(0)}\right)^{m^{j}} \equiv 0 \\
\Leftrightarrow \quad \dot{\alpha}_{j}^{(0)}=\left(\frac{\partial f\left(t, y_{0}^{(0)}(t)\right)}{\partial y} \varphi_{j}(t)-\dot{\varphi}_{j}(t)+\right. \\
\left.+\frac{\left(K(t, t) \varphi_{j}(t)\right)}{\lambda_{j}(t)}, \chi_{j}(t)\right) \alpha_{j}^{(0)}+ \\
+\sum_{m^{j} \in \Gamma_{j},\left|m^{j}\right| \leq N}\left(\tilde{f}^{\left(m^{j}\right)}(t), \chi_{j}(t)\right)\left(\alpha^{(0)}\right)^{m^{j}}, \\
j=\overline{1, n}, t \in[0, T], \overline{(22)} \\
\alpha_{k}^{(0)}(0)=\left(z^{0}+A^{-1}(0) h(0), \chi_{k}(0)\right), k=1, n,
\end{gathered}
$$

A system of equations (22) in relation to the unknown vector function $\alpha^{(0)} \equiv\left(\alpha_{1}^{(0)}, \ldots, \alpha_{n}^{(0)}\right)$ is called normal form (see, e.g., [5]). The system (22) is non-linear, and its solvability in the interval $[0, T]$ (i.e. solvability as a whole on the segment $[0, T]$ ) is not guaranteed by anything. Below are given sufficient conditions for such solubility. We require that the normal form (22) be solvable on $[0, T]$. In this case will be built a unique solution of (18) of the problem $\left(\overline{9}_{0}\right)$. Subsequent problems $\left(\overline{9}_{k}\right)$ when 
$k \geq 2$, will always be solved in general in the interval $[0, T]$, since the function $\hat{P}_{k}\left(t, y_{0}, \ldots, y_{k-1}\right)$ is linear with respect to $y_{k-1}$, which means that the corresponding conditions of orthogonality (11) lead to linear differential equations with respect to arbitrary scalar functions $\alpha_{j}^{(k-1)}(t)$, within the solution $y_{k-1}(t, \tau) \in \hat{U} \quad$ of the problem $\left(\overline{9}_{k}\right)$. Formulate the result obtained in the form of approval.

Theorem 2. Suppose that the conditions 1)-4) are satisfied, the normal form (22) is solvable on the segment $[0, T]$ as a whole. Then all iterative problems $\left(\overline{9}_{k}\right)(k \geq 0) \quad$ during their successive solution uniquely solvable in the space $\hat{U}$.

Let us now study the solvability of nonlinear normal forms (22). Let $t=t^{*}$ is fixed point of the segment $[0, T]$. Values of the eigenvalues $\lambda_{j}(t)$ in the point $t=t^{*}$ will simply designate $\lambda_{j}$ (i.e. $\left.\lambda_{j}\left(t^{*}\right)=\lambda_{j}\right)$. Overlay the numbers $\lambda_{j}, j=\overline{1, n}$ on the complex plane $\lambda$. Then you have the following range of events at the location of the plane $\lambda$.

Case 1: There is a line $(\pi)$, passing through the origin of the complex plane $\lambda$, such that all $\lambda_{j}$ lie on one side of it and there is no point $\lambda_{j}$. In this case, we enumerate the numbers $\lambda_{j}$ so that $0<\mu_{1} \leq \mu_{2} \leq \ldots \leq \mu_{n}$, where $\mu_{j}$ is the distance from point $\lambda_{j}$ to line $(\pi), j=\overline{1, n}$.

Case 2. There is no a line $(\pi)$, as referred to in the case of 1 .

Case 1 means that the convex hull of $\omega\left(\lambda_{1}, \ldots, \lambda_{n}\right)$ of the numbers $\lambda_{j}$ does not contain zero $\lambda=0$, and the case 2 means that $\omega\left(\lambda_{1}, \ldots, \lambda_{n}\right)$ contains zero $\lambda=0$. It turns out that in case 1 the number of resonant exponentials in (22) is finite, and the form (22) itself is triangular, i.e. we have the following proposition (see [2], p. 240-243).

Theorem 3. If the numbers $\lambda_{j}(j=\overline{1, n})$ refer to case 1, they are pairwise distinct (that is, $\lambda_{i} \neq \lambda_{j}, i \neq j, i, j=\overline{1, n}$ ) and are numbered so that $0<\mu_{1} \leq \mu_{2} \leq \ldots \leq \mu_{n}$, then the disjunctive system of equations $\left(m^{j}, \lambda\right)=\lambda_{j},\left|m^{j}\right| \geq 2, j=\overline{1, n}$ relative to unknown multi-indices $m^{j}=\left(m_{1}^{j}, \ldots, m_{n}^{j}\right)$ has only such solutions that are contained among the solutions of the disjunctive system

$$
m_{1}^{2} \lambda_{1}=\lambda_{2}
$$

$$
m_{1}^{3} \lambda_{1}+m_{2}^{3} \lambda_{2}=\lambda_{3},
$$

$$
m_{1}^{n} \lambda_{1}+m_{2}^{n} \lambda_{2}+\ldots+m_{n-1}^{n} \lambda_{n-1}=\lambda_{n},\left|m^{j}\right| \geq 2, j=\overline{2, n} .
$$

In other words, for every fixed $j \in\{1,2, \ldots, n\}$ all solutions of the equation $\left(m^{j}, \lambda\right)=\lambda_{j},\left|m^{j}\right| \geq 2, j=\overline{1, n}$ have the form $m^{j}=\left(m_{1}^{j}, \ldots, m_{j-1}^{j}, 0, \ldots, 0\right)\left(\left|m^{j}\right| \geq 2\right)$.

It follows that system (22) is triangular and polynomial, i.e., (22) has the form

$$
\begin{gathered}
\dot{\alpha}_{1}^{(0)}=p_{1}(t) \alpha_{1}^{(0)}, \quad \alpha_{1}^{(0)}(0)=\bar{\alpha}_{1}^{(0)}, \\
\dot{\alpha}_{2}^{(0)}=p_{2}(t) \alpha_{2}^{(0)}+\tilde{f}_{m_{1}^{2}, 2}(t) \alpha_{1}^{0_{1}^{2}}, \quad \alpha_{2}^{(0)}(0)=\bar{\alpha}_{2}^{(0)}, \\
\ldots \\
\dot{\alpha}_{n}^{(0)}=p_{n}(t) \alpha_{n}^{(0)}+\sum_{2 \leq\left|m^{n}\right| \leq N, m_{1}^{n} \lambda\left(t_{*}\right)+\ldots+} f_{m_{1}^{n}, \ldots, m_{n-1}^{n}}(t) \times \\
\times \alpha_{1}^{0^{m_{1}^{n}}} \ldots \alpha_{n-1}^{m_{n-1}^{n}}, \quad \alpha_{n-1}^{n} \lambda_{n-1}^{(0)}(0)=\bar{\alpha}_{n}^{(0)},
\end{gathered}
$$

where

$$
\begin{gathered}
p_{j}(t) \equiv\left(\frac{\partial f\left(t, y_{0}^{(0)}(t)\right)}{\partial y} \varphi_{j}(t)-\dot{\varphi}_{j}(t)+\right. \\
\left.+\frac{\left.(K) t, t) \varphi_{j}(t)\right)}{\lambda_{j}(t)}, \chi_{j}(t)\right), \\
\bar{\alpha}_{j}^{(0)}=\alpha_{j}^{(0)}(0)=\left(z^{0}+A^{-1}(0) h(0), \chi_{j}(0)\right), j=\overline{1, n} .
\end{gathered}
$$

Each $j$-th equation of this system always has a unique solution $\alpha^{0}=\alpha^{0}(t)$ in the field of a certain continuity of its coefficients, i.e. in the segment $[0, T]$. Belonging the solution $\alpha^{0}(t)$ to the class $\mathrm{C}^{\infty}\left([0, T], \mathrm{C}^{n}\right)$ follows from the infinite differentiability of the right-hand side of system (22) with respect $t$ and $\alpha^{0}$.

Comment. If the eigenvalues $\lambda_{j}=\lambda_{j}\left(t_{*}\right)$ relate to the case 2, the system (22) will not be triangular. However, as shown in [5] (p. 148), in this case, the order of the normal form can be lowered. Thus, the question of the solvability of the generally normal form (22) reduces to the solvability of a simpler differential system. However, to write this system structurally quite difficult, so the question of the solvability of the normal form (22) in the case 2 will not be discussed.

If the conditions of Theorem 2 are satisfied, then we can construct series (6), whose coefficients $y_{k}(t, \tau) \in \hat{U} \quad$ satisfy the iterative problems $\left(\overline{9}_{k}\right), k \geq 0 . \quad$ Let $\left.\quad y_{\varepsilon N}(t) \equiv S_{N}(t, \tau, \varepsilon)\right|_{\tau=\psi(t) / \varepsilon} \equiv$ 
$\left.\equiv \sum_{k=1}^{N} \varepsilon^{k} y_{k}(t, \tau)\right|_{\tau=\psi(t) / \varepsilon}$ is narrowing $N$-th partial sum of this series at $\tau=e^{\frac{\psi(t)}{\varepsilon}}$. The following statement holds, which is proved in the same way as the similar statement in [2], § 4.5.

Theorem 4. Let the conditions of Theorem 2 are satisfied. Then the problem (1) has for sufficiently small $\quad \varepsilon\left(0<\varepsilon \leq \varepsilon_{0}\right) \quad$ unique solution $y(t, \varepsilon) \in \mathrm{C}^{1}\left([0, T], \mathrm{C}^{n}\right)$. and in this case we have the estimate

$$
\left\|y(t, \varepsilon)-y_{\varepsilon N}(t)\right\|_{C[0, T]} \leq c_{N} \varepsilon^{N+1}(N=0,1,2, \ldots),
$$

where $c_{N}>0$ is constant, independent of $\varepsilon$, when $\varepsilon \in\left(0, \varepsilon_{0}\right]\left(\varepsilon_{0}>0\right.$ is small enough).

\section{Examples}

We present examples illustrating features of the above theory.

Example 1. Consider an integro-differential problem

$$
\begin{aligned}
& \varepsilon \frac{d z}{d t}=\lambda_{1} z+\int_{0}^{t}\left[k_{11}(t, s) z(s, \varepsilon)+\right. \\
& \left.+k_{12}(t, s) w(s, \varepsilon)\right] d s+\varepsilon h_{1}(t), z(0, \varepsilon)=y^{0}, \\
& \varepsilon \frac{d w}{d t}=\lambda_{2} w+\int_{0}^{t}\left[k_{21}(t, s) z(s, \varepsilon)+\right. \\
& \left.+k_{22}(t, s) w(s, \varepsilon)\right] d s+\varepsilon z^{2}+\varepsilon h_{2}(t), w(0, \varepsilon)=z^{0},
\end{aligned}
$$

where

$$
\lambda_{2}=2 \lambda_{1} \quad \text { are }
$$

constant,

$\operatorname{Re} \lambda_{j} \leq 0, j=1,2, t \in[0, T]$ (here the role of $y$ played by the vector $y=\{z, w\})$. After the regularization problem (22) by means of variables

$$
\tau_{j}=\frac{1}{\varepsilon} \int_{0}^{t} \lambda_{j} d \theta=\frac{\lambda_{j} t}{\varepsilon} \equiv \frac{\psi_{j}(t)}{\varepsilon}, j=1,2
$$

we arrive at the following extended problem:

$$
\begin{array}{r}
\varepsilon \frac{\partial \tilde{y}}{\partial t}+\sum_{j=1}^{2} \lambda_{j} \frac{\partial \tilde{y}}{\partial \tau_{j}}-\Lambda \tilde{y}-\varepsilon\left(\begin{array}{l}
0 \\
\tilde{z}^{2}
\end{array}\right)-\tilde{J} \tilde{y}=\varepsilon h(t), \\
\left.\tilde{y}(t, \tau, \varepsilon)\right|_{t=0, \tau=0}=y^{0}
\end{array}
$$

where indicated:

$$
\begin{gathered}
\tilde{y}=\{\tilde{z}, \tilde{w}\}, \Lambda=\operatorname{diag}\left(\lambda_{1}, \lambda_{2}\right), h(t)=\left\{h_{1}(t), h_{2}(t)\right\}, \\
y^{0}=\left(z^{0}, w^{0}\right), \tilde{J} \tilde{y}(t, \tau, \varepsilon)=\sum_{r=0}^{\infty} \varepsilon^{r} \sum_{s=0}^{r} R_{r-s} y_{s}(t, \tau), \\
\tilde{y}(t, \tau, \varepsilon)=\sum_{k=0}^{\infty} \varepsilon^{k} y_{k}(t, \tau),
\end{gathered}
$$

and operators $R_{k}$ act in the space $U$ of functions (3) and are calculated according to formulas (see $\left.\left.\left(5_{k}\right)\right)\right)$ :

$$
\begin{gathered}
R_{0} y(t, \tau)=\int_{0}^{t} K(t, s) y_{0}(s) d s, \quad(249) \\
R_{1} y(t, \tau)=\sum_{i=1}^{2}\left[\left(I_{i}^{0}\left(K(t, s) y_{i}(s)\right)\right)_{s=t} e^{\tau_{i}}-\right. \\
\left.-\left(I_{i}^{0}\left(K(t, s) y_{i}(s)\right)\right)_{s=0}\right]+ \\
+\sum_{2 \leq|m| \leq N_{z}}^{*}\left[\left(I_{m}^{0}\left(K(t, s) y^{m}(s)\right)\right)_{s=t} e^{(m, \tau)}-\right. \\
\left.-\left(I_{m}^{0}\left(K(t, s) y^{m}(s)\right)\right)_{s=0}\right], \\
I_{i}^{0}=\frac{1}{\lambda_{i}(s)} \cdot I_{i}^{v}=\frac{1}{\lambda_{i}(s)} I_{i}^{v-1}(v \geq 1, i=1,2), \\
I_{m}^{0}=\frac{1}{(m, \lambda(s))} \cdot I_{m}^{v}=\frac{1}{(m, \lambda(s))} \frac{\partial}{\partial s} I_{m}^{v-1} \quad(v \geq 1,|m| \geq 2), \\
\text { where } \quad K(t, s)=\left(\begin{array}{ll}
k_{11}(t, s) & k_{12}(t, s) \\
k_{21}(t, s) & k_{22}(t, s)
\end{array}\right) \quad(\text { when } \quad k \geq 2
\end{gathered}
$$

operators $R_{k}$ do not write). For the coefficients $y_{k}(t, \tau)$ of the series (24) we obtain the iterative problem (see $\left(9_{k}\right)$ ), right-hand sides of which can not belong to the space $U$. Therefore, they need an embedding to the space $U$, and we get the following problems:

$$
\begin{array}{r}
\mathrm{L} y_{0}(t, \tau) \equiv \sum_{j=1}^{2} \lambda_{j} \frac{\partial y_{0}}{\partial \tau_{j}}-\Lambda y_{0}-R_{0} y_{0}=0, \\
y_{0}(0,0)=y^{0} ; \\
\mathrm{L} y_{1}(t, \tau)=-\frac{\partial y_{0}}{\partial t}+\left(\begin{array}{l}
0 \\
z_{0}^{2}
\end{array}\right)^{\wedge}+R_{1} y_{0}+h(t), \\
y_{1}(0,0)=0 ; \\
\mathrm{L} y_{2}(t, \tau)=-\frac{\partial y_{1}}{\partial t}+\left(\begin{array}{l}
0 \\
z_{0} z_{1}
\end{array}\right) \wedge+R_{1} y_{1}+ \\
+R_{2} y_{0}, y_{2}(0,0)=0 ;
\end{array}
$$

$$
\begin{aligned}
& \mathrm{L} y_{k}(t, \tau)=-\frac{\partial y_{k-1}}{\partial t}+\hat{P}_{k}\left(y_{0}, \ldots, y_{k-1}, t\right)+ \\
& +R_{k} y_{0}+\ldots+R_{1} y_{k-1}, y_{k}(0,0)=0, k \geq 3
\end{aligned}
$$

The problem $\left(\overline{25}_{0}\right)$ has the solution in the space $U$ :

$$
z_{0}(t, \tau)=\alpha_{1}(t) e^{\tau_{1}}, w_{0}(t, \tau)=\alpha_{2}(t) e^{\tau_{2}},
$$

where $\quad \alpha_{j}(t) \in C^{\infty}\left([0, T], \mathrm{C}^{1}\right)$ are arbitrary functions. Subjecting the initial condition $y_{0}(0,0)=y^{0}$, we find values $\alpha_{1}(0)=z^{0}, \alpha_{2}(0)=w^{0}$. For the final calculation of the functions $\alpha_{j}(t)$, we pass to the next system $\left(\overline{25}_{1}\right)$ : 


$$
\begin{gathered}
\mathrm{L} y_{1}(t, \tau)=-\frac{\partial}{\partial t}\left(\begin{array}{l}
\alpha_{1}(t) e^{\tau_{1}} \\
\alpha_{2}(t) e^{\tau_{2}}
\end{array}\right)+\left(\begin{array}{l}
0 \\
\alpha_{1}^{2}(t) e^{2 \tau_{1}}
\end{array}\right) \wedge h(t)+ \\
\left.+\sum_{j=1}^{2} \mid \frac{\left(K(t, t) \alpha_{j}(t) e_{1}\right)}{\lambda_{j}(t)} e^{\tau_{j}}-\frac{\left(K(t, 0) \alpha_{j}(0) e_{2}\right)}{\lambda_{j}(0)}\right] \equiv \\
\equiv-\frac{\partial}{\partial t}\left(\begin{array}{l}
\alpha_{1}(t) e^{\tau_{1}} \\
\alpha_{2}(t) e^{\tau_{2}}
\end{array}\right)+\left(\begin{array}{c}
0 \\
\alpha_{1}^{2}(t) e^{\tau_{2}}
\end{array}\right)+\frac{1}{\lambda_{1}}\left(\begin{array}{l}
k_{11}(t) \\
k_{21}(t)
\end{array}\right) \alpha_{1}(t) e^{\tau_{1}}+ \\
+\frac{1}{\lambda_{2}}\left(\begin{array}{l}
k_{21}(t) \\
k_{22}(t)
\end{array}\right) \alpha_{2}(t) e^{\tau_{2}}-\frac{1}{\lambda_{1}}\left(\begin{array}{l}
k_{11}(0) \\
k_{21}(0)
\end{array}\right) \alpha_{1}(0)- \\
-\frac{1}{\lambda_{2}}\left(\begin{array}{l}
k_{21}(0) \\
k_{22}(0)
\end{array}\right) \alpha_{2}(0)+\left(\begin{array}{l}
h_{1}(t) \\
h_{2}(t)
\end{array}\right)
\end{gathered}
$$

where $e_{1}=\{1,0\}, e_{2}=\{0,1\}$. This system is solvable in the space $U$ if and only if

$$
\begin{gathered}
\dot{\alpha}_{1}(t)-\frac{k_{11}(t)}{\lambda_{1}} \alpha_{1}(t) \equiv 0, . \\
\dot{\alpha}_{2}(t)-\frac{k_{22}(t)}{\lambda_{2}} \alpha_{2}(t)+\alpha_{1}^{2}(t) \equiv 0 .
\end{gathered}
$$

Attaching the initial conditions $\alpha_{1}(0)=z^{0}, \alpha_{2}(0)=w^{0}$ to these identities we find unique functions $\alpha_{j}(t)$ :

$$
\begin{gathered}
\alpha_{1}(t)=z^{0} e^{\int_{0}^{t} \frac{k_{11}(\theta)}{\lambda_{1}} d \theta}, \\
\alpha_{2}(t)=e^{\int_{0}^{t} \frac{k_{22}(\theta)}{\lambda_{2}} d \theta}\left(w^{0}+\int_{0}^{t} e^{-\int_{0}^{s_{k 22}} \frac{k_{2}(\theta)}{\lambda_{2}} d \theta} \alpha_{1}^{2}(\theta) d s\right) .
\end{gathered}
$$

In this case the principal term of asymptotics of the solution of the problem (22) will be:

$$
z_{\varepsilon 0}(t)=\alpha_{1}(t) e^{\frac{\lambda_{1} t}{\varepsilon}}, w_{\varepsilon 0}(t)=\alpha_{2}(t) e^{\frac{\lambda_{2} t}{\varepsilon}} .
$$

This shows that the nonlinearity of $f(y, t)=\left\{0, z^{2}\right\}$ affects the leading term of the asymptotics.

Example 2. Consider now the integrodifferential problem

$$
\begin{aligned}
& \varepsilon \frac{d z}{d t}=-i_{1} z+\int_{0}^{t}\left[k_{11}(t, s) z(s, \varepsilon)+\right. \\
& \left.+k_{12}(t, s) w(s, \varepsilon)\right] d s+\varepsilon z^{2} w+\varepsilon h_{1}(t), z(0, \varepsilon)=y^{0}, \\
& \varepsilon \frac{d w}{d t}=i w+\int_{0}^{t}\left[k_{21}(t, s) z(s, \varepsilon)+\right. \\
& \left.+k_{22}(t, s) w(s, \varepsilon)\right] d s+\varepsilon h_{2}(t), w(0, \varepsilon)=z^{0},
\end{aligned}
$$

Here the eigenvalues $\lambda_{1}=-i, \lambda_{2}=+i$ refer to case 2, and the sets of resonant multiindices have the form

$$
\begin{gathered}
\Gamma_{1}=\left\{\left(m_{1}^{1}, m_{2}^{1}\right)=(n+1, n), n \in \mathrm{N}\right\}, \\
\Gamma_{2}=\left\{\left(m_{1}^{2}, m_{2}^{2}\right)=(n, n+1), n \in \mathrm{N}\right\},
\end{gathered}
$$

$$
\Gamma_{0}=\left\{\left(m_{1}, m_{2}\right)=(n, n), n \in \mathrm{N}\right\} .
$$

Introducing the regularizing variables

$$
\tau_{j}=\frac{(-1)^{j} i \cdot t}{\varepsilon} \equiv \frac{\psi_{j}(t)}{\varepsilon}, j=1,2,
$$

and regularizing the integrals in the space $U$ and the subsequent embedding operation, we obtain the following iterative problems:

$$
\begin{aligned}
& \mathrm{L} y_{0}(t, \tau) \equiv \sum_{j=1}^{2} \lambda_{j} \frac{\partial y_{0}}{\partial \tau_{j}}-\Lambda y_{0}-R_{0} y_{0}=0, \\
& y_{0}(0,0)=y^{0} ; \\
& \mathrm{L} y_{1}(t, \tau)=-\frac{\partial y_{0}}{\partial t}+\left(\begin{array}{l}
z_{0}^{2} w \\
0
\end{array}\right) \wedge+R_{1} y_{0}+h(t), \\
& y_{1}(0,0)=0 ; \\
& \begin{array}{r}
\mathrm{L} y_{k}(t, \tau)=-\frac{\partial y_{k-1}}{\partial t}+\hat{P}_{k}\left(y_{0}, \ldots, y_{k-1}, t\right)+ \\
+R_{k} y_{0}+\ldots+R_{1} y_{k-1}, y_{k}(0,0)=0, k \geq 3,
\end{array}
\end{aligned}
$$

where, as in the previous example, it is indicated: $\Lambda=\operatorname{diag}(-i,+i), y=(z, w), h(t)=\left\{h_{1}, h_{2}\right\}$. The first iteration problem $\left(\overline{27}_{0}\right)$ has a solution in the form of vector functions (26), where $\alpha_{j}(t) \in C^{\infty}\left([0, T], \mathrm{C}^{1}\right)$ are while arbitrary functions. Subjecting (26) the initial condition $y_{0}(0,0)=y^{0}$, we find the values $\alpha_{1}(0)=z^{0}, \alpha_{2}(0)=w^{0}$. For the final evaluation of the functions $\alpha_{j}(t)$ it is necessary to go to the next iteration system $\left(\overline{27}_{1}\right)$ :

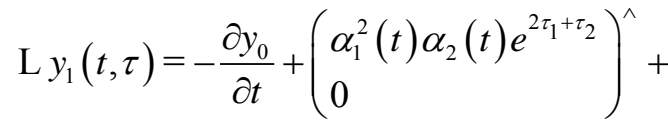

$$
\begin{aligned}
& +R_{1} y_{0}+h(t) \Leftrightarrow \mathrm{L} y_{1}(t, \tau)=-\frac{\partial}{\partial t}\left(\begin{array}{c}
\alpha_{1}(t) e^{\tau_{1}} \\
\alpha_{2}(t) e^{\tau_{2}}
\end{array}\right)+ \\
& +\left(\begin{array}{l}
\alpha_{1}^{2}(t) \alpha_{2}(t) e^{\tau_{1}} \\
0
\end{array}\right)+\frac{1}{\lambda_{1}}\left(\begin{array}{l}
k_{11}(t) \\
k_{21}(t)
\end{array}\right) \alpha_{1}(t) e^{\tau_{1}}+ \\
& +\frac{1}{\lambda_{2}}\left(\begin{array}{l}
k_{21}(t) \\
k_{22}(t)
\end{array}\right) \alpha_{2}(t) e^{\tau_{2}}-\frac{1}{\lambda_{1}}\left(\begin{array}{l}
k_{11}(0) \\
k_{21}(0)
\end{array}\right) \alpha_{1}(0)- \\
& -\frac{1}{\lambda_{2}}\left(\begin{array}{l}
k_{21}(0) \\
k_{22}(0)
\end{array}\right) \alpha_{2}(0)+\left(\begin{array}{l}
h_{1}(t) \\
h_{2}(t)
\end{array}\right) .
\end{aligned}
$$

Orthogonality conditions (9) lead to a system of equations

$$
\left\{\begin{array}{lc}
\dot{\alpha}_{1}(t)-\alpha_{1}^{2}(t) \alpha_{2}(t)-\frac{k_{11}(t)}{\lambda_{1}} \alpha_{1}(t)=0, & \alpha_{1}(0)=z^{0}, \\
\dot{\alpha}_{2}(t)-\frac{k_{22}(t)}{\lambda_{2}} \alpha_{2}(t)=0, & \alpha_{2}(0)=w^{0},
\end{array} \Leftrightarrow\right.
$$




$$
\Leftrightarrow\left\{\begin{array}{c}
\alpha_{2}(t)=w^{0} e^{\frac{t}{0} \frac{k_{22}(\theta)}{\lambda_{2}} d \theta}, \\
\dot{\alpha}_{1}(t)-\frac{k_{11}(t)}{\lambda_{1}} \alpha_{1}(t)-\alpha_{2}(t) \alpha_{1}^{2}(t)=0, \quad \alpha_{1}(0)=z^{0} .
\end{array}\right.
$$

The second equation of this system has the following solution:

$$
\alpha_{1}(t)=\frac{z^{0} e^{\int^{t} \frac{k_{11}(x)}{\lambda_{1}} d x}}{1-\left(\int_{0}^{t}\left(e^{\int^{s} \frac{k_{11}(x)}{\lambda_{1}} d x} \alpha_{2}(s)\right) d s\right) z^{0}=0} .
$$

This solution $[0, T]$, does not exist on the interval if there is $t_{0} \in[0, T]$ that

$$
1-\left(\int_{0}^{t}\left(e^{\int^{s} \frac{k_{11}(x)}{\lambda_{1}} d x} \alpha_{2}(s)\right) d s\right) z^{0}=0 .
$$

In this case, it is impossible to construct the asymptotic solution of the original problem (27).

Note that in the previous and in this example, the zero point of the spectrum does not participate in the construction of the leading term of the asymptotics.

However, if we take, for example, a nonlinearity of the iterative problem $\left(\overline{27}_{1}\right)$ will arise resonance exponentials $e^{(m, \tau)}$ with multi-indexes $m \in \Gamma_{0}$ and their embedding in the space $U$ will affect the construction of the main term of solutions asymptotic term (27).

\section{References:}

1. Lomov, S.A., Introduction to General Theory of Singular Perturbations, vol. 112 of Translations of Math. Monographs, American Math. Society, Providence, USA, 1992.

2. Safonov, V.F., Bobodzhanov, A.A., Course of higher mathematics. Singularly perturbed equations and the regularization method: textbook, Moscow, Publishing House of MPEI, 2012. (in Russian)

3. Lomov, S.A., Lomov, I.S., Foundations of mathemathical theory of boundary layer, Moscow, Publishing House of MSU, 2011. (in Russian)

4. Bobodzhanov, A.A., Safonov, V.F., Volterra integral equations with rapidly changing kernels and their asymptotic integration, Math. collection, Vol. 192. No 8, 2011, pp. 53-78.

5. Bryuno, A.D., The analytical form of differential equations, Proceed. Moscow Math. Society, Vol. 25, 1971, pp. 119-262. (In Russian)

\section{Creative Commons Attribution License 4.0 (Attribution 4.0 International, CC BY 4.0)}

This article is published under the terms of the Creative Commons Attribution License 4.0 https://creativecommons.org/licenses/by/4.0/deed.en US 